\title{
La ardilla de vientre rojo en Argentina y el manejo de especies introducidas como un problema biológico y social
}

\author{
Mariela Borgnia; Verónica Benitez; Cecilia Gozzi \& M. Laura Guichón ${ }^{\bowtie}$ \\ Ecología de Mamíferos Introducidos (EMI), Depto. Ciencias Básicas, Universidad Nacional de Luján.
}

\begin{abstract}
RESUMEN. El estudio y manejo de especies exóticas invasoras ha estado principalmente enfocado en conocer el proceso de invasión y técnicas adecuadas de manejo. Sin embargo, en los últimos años los estudios que promueven la participación de diferentes grupos sociales de la comunidad en los planes de manejo han cobrado importancia. En este trabajo describimos las opiniones y actitudes predominantes de seis grupos sociales (productores y prestadores de servicios, ambientalistas, vecinos, personal de áreas protegidas, funcionarios y docentes) con respecto a la presencia de la ardilla de vientre rojo introducida en Argentina. La existencia de varios focos de invasión de ardillas en tres provincias argentinas se relaciona con liberaciones intencionales de ardillas, en ocasiones obtenidas por comercio ilegal. Las opiniones con respecto a la presencia de ardillas variaron desde una percepción negativa que consideraba que era una especie problema que debía ser controlada hasta una percepción positiva que sostiene que es una especie atractiva y vistosa. Algunos grupos mostraron una opinión predominante, mientras que otros reflejaron todo el rango de respuestas. La experiencia directa con la especie, sus atributos y el tiempo desde su introducción, el área de residencia y el grado de conocimiento sobre la problemática influyen sobre las opiniones y actitudes de las personas. Las alternativas de manejo deben planificarse para cada zona invadida teniendo en cuenta las particularidades no sólo biológicas y económicas, sino también sociales y políticas. Al mismo tiempo, resulta imprescindible una estrategia nacional de prevención para frenar la aparición de nuevos focos de invasión. Para esto es fundamental desarrollar estrategias que reorienten la relación de las personas con los recursos del ambiente y promuevan la valoración de los ecosistemas locales y especies nativas, así como la co-responsabilidad y la participación ciudadana en la problemática de las invasiones biológicas, el tráfico de fauna y la tenencia de mascotas.
\end{abstract}

[Palabras clave: especie carismática, invasiones biológicas, percepción ambiental]

\begin{abstract}
Aвstract. The red-bellied squirrel in Argentina and the management of introduced species as a biological and social problem: The study and management of exotic invasive species has been mainly focused on understanding the invasion process and designing adequate management techniques; however, in recent years several studies have shown the importance of involving social groups of the local community in the implementation of management plans. In this study we describe opinions and attitudes of six social groups (producers and service providers, environmentalists, neighbours, personnel of protected areas, civil servants, and teachers) regarding the presence of the red-bellied squirrels introduced in Argentina. The occurrence of various invasion foci of squirrels in three Argentinian provinces results from intentional releases of individuals, sometimes obtained by illegal trade. Opinion towards the presence of squirrels ranged from a negative perception of the squirrels viewed as a problem species that needs to be controlled to a positive perception of the squirrels viewed as an attractive species. Some groups showed a predominant opinion while others reflected the whole range of responses. Personal experience with the species, its attributes and the time since its introduction, the area of residency, and the knowledge of the problems posed by this species affect the opinion and attitudes towards the presence of red-bellied squirrels. Management plans must be designed for each invasion focus not only considering biological and economic issues, but also social and political ones. At the same time, a national strategy of prevention to stop the creation of new invasion foci is urgently needed. We therefore need strategies to reorient the relationship between people and environmental resources and to promote appreciation of local ecosystems and native species as much as co-responsibility and citizen participation in the problems posed by biological invasions, and the illegal trade of wild fauna and pets.
\end{abstract}

[Keywords: charismatic species, biological invasions, environmental perceptions]

\section{ESPECIES EXÓTICAS INVASORAS Y LAS ARDILLAS INTRODUCIDAS EN ARGENTINA}

Desde hace años, las especies exóticas invasoras (EEI) son el foco de muchos estudios y debates debido a los impactos que tienen

$\triangle$ Instituto de Investigaciones en Biodiversidad y Medioambiente (INIBIOMA-CONICET) sede Junín de los Andes, Neuquén.

ardillas@unlu.edu.ar sobre la biodiversidad, sobre la modificación de ecosistemas y sobre aspectos económicos y socio-culturales en áreas urbanas y rurales. Estos estudios y debates surgen debido a las consecuencias de dichos impactos en las actividades productivas, a los daños a la

Recibido: 10 de febrero de 2013; Fin de arbitraje: 25 de mayo; Nueva versión: 17 de julio; Aceptado: 14 de agosto. 
infraestructura y los servicios urbanos, y al costo de implementar acciones de manejo (IUCN 2000; Mack et al. 2000; Simberloff 2000; Pimentel et al. 2005). Existen numerosos casos en que las especies invasoras han tenido efectos notorios y drásticos. Sin embargo, el impacto puede ser muy variable en intensidad y tipo, y puede llevar varios años hasta que se hace evidente (Simberloff et al. 2013). Además, debe tenerse en cuenta que la valoración del impacto depende de los intereses en cuestión ya que, por ejemplo, una especie invasora puede ser beneficiosa desde el punto de vista productivo pero tener un impacto negativo desde el punto vista de ambiental.

El manejo de las especies introducidas que se convierten en invasoras es complejo, en parte debido a que el ser humano está involucrado en todo el proceso. Por un lado, es el vector que favorece las introducciones (accidentales e intencionales), ya sea por razones económicas o por motivos ornamentales. Por otro lado, es el destinatario de gran parte de los problemas ocasionados por las EEI, y a su vez es el responsable de decidir e implementar las acciones de manejo (García Llorente et al. 2008). A la diversidad de los procesos biológicos en cada invasión se suma la gran diversidad de percepciones, opiniones y actitudes de distintos sectores de la sociedad, vinculados en mayor o menor medida con aspectos comunicacionales, de opinión pública, cosmovisión individual, investigación y gestión (Glaves 2009; Simberloff et al. 2013). La información sobre cuáles son EEI, los problemas que causan y los métodos posibles de control suele ser insuficiente o inadecuada, por lo que pocas personas perciben que están siendo afectadas negativamente por EEI, ya sea de forma directa o indirecta (Lodge \& Shrader-Frechette 2003; Lundberg 2010). En ocasiones, el entendimiento entre diferentes personas o grupos se dificulta frente al uso de un lenguaje emotivo y manipulativo acerca de las EEI, tanto por parte de los científicos como de la comunidad en general (Simberloff 2003; Larson 2005; McNeely 2011).

Más allá de aspectos comunicacionales o de información, existen diferentes opiniones entre personas pertenecientes a distintos grupos sociales (como ser gestores, ambientalistas, productores, turistas, científicos) debido a los intereses y objetivos de cada grupo y a las distintas concepciones o marco ético desde el cual se mira el mundo natural. Algunos muestran un enfoque ecocentrista, enfocado en los ecosistemas y procesos, según el cual las invasiones deberían ser evitadas $\mathrm{y}$, de ser posible, las EEI deberían ser erradicadas para proteger la biodiversidad. Mientras tanto, otros tienen un enfoque centrado en el Hombre, antropocentrista, en el cual el impacto ecológico no es importante a menos que traiga aparejado daños sociales o económicos. También pueden adherir al zoocentrismo, enfocado sobre el bienestar de los animales; en este enfoque prima un desacuerdo con los métodos de control que involucren el sacrificio de animales, aunque sea en beneficio de individuos de otras especies (Salazar Barragan \& Serna Mendoza 2006). En otros casos, las posturas de personas o grupos se identifican con otra clasificación de la naturaleza (Kellert 1996), en la que se definen las posturas utilitaria (naturaleza como recurso, fuente de utilidad material y física), naturalista (explorar y descubrir la naturaleza), ecológico-científica (conocer y entender la naturaleza), estética (atracción física y placentera hacia la naturaleza), simbólica (naturaleza como referencia del lenguaje y la comunicación), dominante (control y poder sobre la naturaleza), humanista (liga emocional con la naturaleza), moralista (relación ética y espiritual con la naturaleza) o negativista (temor y aversión a la naturaleza). Estas distintas valoraciones y posturas afectan la percepción y actitud en relación al impacto (negativo, nulo o positivo) de una especie en particular, algo que ha sido subestimado en gran medida por los estudios científicos y planes de manejo de EEI (Lodge \& Shrader-Frechette 2003).

Bajo este marco conceptual desarrollamos nuestro estudio relacionado con la problemática social que despierta la expansión de ardillas introducidas en Argentina y los posibles planes de manejo para controlar la invasión. La ardilla de vientre rojo (Callosciurus erythraeus) es un roedor silvestre, de hábitos diurnos y arborícolas, originario del sudeste de Asia, que fue introducido en Argentina en 1970 con fines ornamentales (Aprile \& Chicco 1999). Menos de 10 individuos dieron origen a la primera población silvestre en el partido de Luján, provincia de Buenos Aires, que actualmente posee densidades elevadas y continúa en expansión (Guichón \& Doncaster 2008; Benitez et al. 2013). Existen varias poblaciones de ardillas introducidas en Buenos Aires, Córdoba y Santa Fe debido al atractivo que despiertan en muchas personas 
como especie ornamental o mascota (Benítez et al. 2013). Este es un ejemplo de una especie introducida que se vuelve invasora no sólo por sus características biológicas sino también por conductas sociales y actividades ilegales como la captura y liberación de ardillas en nuevas áreas, y su comercio. La motivación que lleva a la introducción de ardillas se relaciona con el concepto de enriquecer la fauna regional sumando una especie vistosa o tenerla como mascota en estancias o sitios parquizados o turísticos, generalmente vinculados a emprendimientos privados o familias adineradas. Más allá de su aspecto carismático, las denuncias de residentes y productores, y las investigaciones realizadas permiten describir algunos daños causados por la ardilla de vientre rojo en sistemas naturales, sistemas productivos y áreas urbanizadas [e.g., el descortezamiento a los árboles, el consumo de frutos y semillas, y en menor medida de flores y brotes, la dispersión de vegetación exótica, el daño a mangueras de riego, cables de telefonía y televisión, el posible impacto sobre algunas aves, el riesgo de transmisión de parásitos y zoonosis, y el riesgo que lleguen a sitios con alto valor de conservación como el Delta del Río Paraná o a ambientes habitados por ardillas nativas (Guichón et al. 2005; Bobadilla et al. 2012; Messetta 2012; Benitez et al. 2013; Gozzi et al. 2013a,b)].

En este trabajo planteamos las siguientes preguntas sobre la presencia de la ardilla de vientre rojo en Argentina: ¿existen diferentes percepciones y actitudes respecto de la situación de esta especie? ¿Qué factores influyen? ¿Qué consecuencias trae respecto a la posibilidad de manejo de la especie? Para responder estas preguntas describimos a continuación una caracterización cualitativa de las percepciones y actitudes de distintos grupos sociales respecto a esta especie y a posibles acciones de manejo.

\section{RECOPILACIÓN DE INFORMACIÓN}

Como iniciativa de nuestro grupo de investigación de la Universidad Nacional de Luján, entre los años 2004 y 2012 desarrollamos actividades de investigación, difusión, educación y gestión. Recopilamos información sobre la opinión respecto de la presencia de ardillas introducidas mediante: 1) entrevistas no estructuradas realizadas a residentes de sitios invadidos por ardillas o cercanos al borde de distribución, 2) talleres convocados para docentes, productores, prestadores de servicio, personal de áreas protegidas, y organizaciones no gubernamentales (ONGs), 3) reuniones técnicas con organismos de gestión, 4) charlas-debate realizadas en escuelas, principalmente destinadas a alumnos de 10-15 años, y 5) comentarios recibidos por correo electrónico o entrevistas con personas que nos contactaron. En los talleres, charlas y reuniones se realizaban exposiciones seguidas de intercambio y debate, y se entregaba material sobre invasiones biológicas, su relación con el tráfico de fauna y la tenencia responsable de mascotas, mostrando a la ardilla como ejemplo regional.

En los talleres, charlas y reuniones se realizaban exposiciones seguidas de intercambio y debate, y se entregaba material sobre invasiones biológicas, su relación con el tráfico de fauna y la tenencia responsable de mascotas, mostrando a la ardilla como ejemplo regional.

Se registraron las opiniones expresadas verbalmente o por escrito de las personas pertenecientes a los siguientes grupos: 1) Productores y prestadores de servicios: este grupo incluye las opiniones de 57 personas, tanto productores propiamente dichos, principalmente deespecies forestales ofrutales, como también referentes, técnicos o asesores de grupos de productores, y personal del Instituto Nacional de Tecnología Agropecuaria (INTA). Además incluye a empleados y autoridades de empresas prestadoras de servicios urbanos, como cooperativas telefónicas. Realizamos cuatro talleres y reuniones informativas en las ciudades de Luján, Mercedes, y 25 de Mayo, y en el INTA Delta, todas en la provincia de Buenos Aires, y también recibimos opiniones por correo electrónico. 2) Ambientalistas: abarca a personas particulares o pertenecientes a organizaciones protectoras de animales $u$ ONG's ambientalistas que opinaron en talleres o sitios de Internet (como blogs, facebook) específicamente sobre este tema, y también algunas cartas enviadas por correo electrónico a nuestro grupo $(n=10)$. No se incluyen los cientos de personas que participaron con comentarios o adhesiones en los sitios de Internet vinculados con este tema. 3) Vecinos: residentes de los sitios donde la ardilla está establecida y alrededores, en los focos de Luján, Escobar, 25 de Mayo y Bella Vista en la provincia de Buenos Aires, de Cañada 
Tabla 1. Síntesis cualitativa de la información obtenida que refleja la postura predominante en los seis grupos sociales respecto de la ardilla de vientre rojo introducida en Argentina. ${ }^{1}$ Fuente: Kellert 1996; Salazar Barragan \& Serna Mendoza 2006.

Table 1. Brief qualitative outline that reflects the dominant opinions and attitudes in the six social groups in relation to the red-bellied squirrels introduced in Argentina. ${ }^{1}$ Source: Kellert 1996; Salazar Barragan \& Serna Mendoza 2006.

\begin{tabular}{|c|c|c|c|c|}
\hline Grupo social & Percepción & Cosmovisión ${ }^{1}$ & Prevención de nuevos focos & Manejo de poblaciones \\
\hline $\begin{array}{l}\text { Productores y } \\
\text { prestadores de } \\
\text { servicios }\end{array}$ & $\begin{array}{l}\text { Negativa, especie } \\
\text { problema }\end{array}$ & $\begin{array}{l}\text { Valoración dominante } \\
\text { de la especie; enfoque } \\
\text { antropocéntrico }\end{array}$ & $\begin{array}{l}\text { Poco interés, prioridad } \\
\text { acciones donde ya están } \\
\text { establecidas }\end{array}$ & $\begin{array}{l}\text { Acciones individuales y } \\
\text { aisladas, reclamo de plan } \\
\text { de control urgente }\end{array}$ \\
\hline Ambientalistas & $\begin{array}{l}\text { Positiva, especie } \\
\text { atractiva }\end{array}$ & $\begin{array}{l}\text { Valoración moralista y } \\
\text { humanista de la especie; } \\
\text { enfoque zoocentrista }\end{array}$ & $\begin{array}{l}\text { Apoyo a impedir comercio y } \\
\text { traslado pero hay propuestas } \\
\text { contraproducentes }\end{array}$ & $\begin{array}{l}\text { Campañas en contra de } \\
\text { control que involucre } \\
\text { sacrificio de animales }\end{array}$ \\
\hline Vecinos & $\begin{array}{l}\text { Todo el rango: } \\
\text { negativa, } \\
\text { indiferente, } \\
\text { positiva }\end{array}$ & $\begin{array}{l}\text { Todo el rango de } \\
\text { valoraciones y enfoques }\end{array}$ & $\begin{array}{l}\text { Difusión "boca en boca" del } \\
\text { problema o mediante blogs } \\
\text { personales }\end{array}$ & $\begin{array}{l}\text { Todo el rango, desde } \\
\text { control individual sin } \\
\text { autorización a oposición } \\
\text { al control }\end{array}$ \\
\hline $\begin{array}{l}\text { Personal de } \\
\text { áreas protegidas }\end{array}$ & $\begin{array}{l}\text { Negativa, especie } \\
\text { problema }\end{array}$ & $\begin{array}{l}\text { Valoración ecológica- } \\
\text { científica del problema; } \\
\text { enfoque ecocéntrico }\end{array}$ & $\begin{array}{l}\text { Difusión mediante charlas } \\
\text { o cartelería, colaboración en } \\
\text { monitoreos }\end{array}$ & $\begin{array}{l}\text { Apoyo a plan de control } \\
\text { autorizado }\end{array}$ \\
\hline Funcionarios & $\begin{array}{l}\text { Negativa, especie } \\
\text { problema }\end{array}$ & $\begin{array}{l}\text { Apertura a un enfoque } \\
\text { ecocéntrico }\end{array}$ & $\begin{array}{l}\text { Actualización de legislación } \\
\text { pertinente, falencia en } \\
\text { fiscalización y difusión }\end{array}$ & $\begin{array}{l}\text { Apoyo parcial, falencia } \\
\text { en decisión política y } \\
\text { asignación de recursos y } \\
\text { personal }\end{array}$ \\
\hline Docentes & $\begin{array}{l}\text { Negativa o } \\
\text { positiva }\end{array}$ & $\begin{array}{l}\text { Todo el rango de } \\
\text { valoraciones; apertura a } \\
\text { un enfoque ecocéntrico }\end{array}$ & $\begin{array}{l}\text { Efecto multiplicador } \\
\text { mediante actividades } \\
\text { educativas }\end{array}$ & $\begin{array}{l}\text { Todo el rango desde } \\
\text { control individual a } \\
\text { oposición al control }\end{array}$ \\
\hline
\end{tabular}

de Gómez en Santa Fe y La Cumbrecita en Córdoba. Son opiniones recabadas principalmente en entrevistas no estructuradas con residentes para averiguar sobre el avistaje de ardillas en su lugar de residencia $(n=192) y$, en menor medida, personas que se contactaron con nuestro grupo $(n=10)$. 4) Personal de áreas protegidas: profesionales, técnicos, voluntarios y empleados que trabajan en áreas protegidas u organizaciones vinculadas como la Reserva Natural Otamendi, Reserva del Pilar, Reserva Los Robles, Administración de Parques Nacionales, Asociación Patrimonio Natural, Asociación para la Conservación de la Naturaleza y Aves Argentinas $(n=20)$. Las opiniones se expresaron en dos talleres y una charla informativa que realizamos en la Universidad Nacional de Luján, la Reserva Natural Otamendi y el Concejo Deliberante de Pilar junto con la Reserva del Pilar, provincia de Buenos Aires. 5) Funcionarios: autoridades y empleados de entidades nacionales, provinciales y municipales relacionadas a organismos de fauna, medioambiente, o producción agrícola $(n=15)$ como la Dirección de Fauna Silvestre de la Nación, Dirección de Fauna y Flora de la Provincia de Buenos Aires, Organismo Provincial de Desarrollo Sustentable (Buenos Aires), Subsecretaría de Sanidad Vegetal (Buenos Aires), Dirección General de Manejo de Fauna y Flora Silvestre (Santa Fe), Agencia Córdoba Ambiente
(Córdoba), y direcciones o secretarías de medio ambiente, producción o zoonosis de varios municipios: Luján, Mercedes, General Rodríguez, San Andrés de Giles, Pilar, Escobar, 25 de Mayo (todos en la provincia de Buenos Aires), Cañada de Gómez (Santa Fe) y La Cumbrecita (Córdoba). 6) Docentes: incluye la opinión de docentes y directivos $(\mathrm{n}=$ 35) de escuelas primarias, medias y terciarios. Las opiniones se recopilaron en tres talleres realizados en Luján y General Rodríguez y en charlas informativas realizadas en la Universidad Nacional de Luján y en instituciones educativas de los partidos de Luján, General Rodríguez, San Andrés de Giles y Escobar de las que participaron 80 establecimientos ubicados en dos focos de invasión en la provincia de Buenos Aires.

\section{OPINIONES Y ACTITUDES}

Analizamos la información obtenida en entrevistas, talleres, reuniones, charlas y cartas para describir cualitativamente las opiniones, percepciones y actitudes registradas con mayor frecuencia dentro de cada grupo (Tabla 1).

\section{Productores y prestadores de servicios}

Todos los miembros de este grupo $(n=57)$ se manifestaron a favor de implementar algún método de control y perciben a la ardilla de 
manera negativa por considerarla una "especie problema" (enfoque antropocéntrico con una valoración dominante de la especie). Se evalúa la pérdida económica y el costo del control y se prioriza la rentabilidad individual. Si bien hay preocupación y estado de alerta respecto de la presencia de ardillas, por lo general no se logra una detección temprana de los daños porque los productores desconocen que el daño observado fue causado por ardillas o porque minimizan el problema. Al incrementar la densidad de ardillas y los daños, inician acciones de control por su cuenta $(n=10)$. En general, desconocen la legislación respecto a las especies silvestres y son escasos los pedidos de autorización y asesoramiento a las autoridades, mientras que algunos recurren a los investigadores. Ya sea por desconfianza en los organismos de gestión, desconocimiento, negligencia o practicidad, se usan métodos de control de ardillas poco recomendables como venenos y armas de fuego. No se realiza una cuantificación económica del daño causado por ardillas, ya sea por falta de recursos o por considerar que no es información necesaria para implementar acciones de control. No muestran interés en frenar la expansión de ardillas con excepción de productores de zonas donde aún no se han establecido. En resumen, perciben a las ardillas como una plaga voraz y manifiestan la necesidad de actuar urgente aún sin contar con autorización o nuevos estudios.

\section{Ambientalistas}

En este grupo la mayoría tiene una visión positiva de la presencia de ardillas $(n=8)$ y la consideran una "especie atractiva" ya que demuestran afinidad por una valoración moralista y humanista hacia la especie (enfoque zoocentrista). Si bien algunos no perciben la presencia de ardillas como algo positivo $(n=2)$, el denominador común de este grupo es que se manifiesta una oposición rotunda a métodos de control que involucren sacrificio de individuos, si bien en algunos casos podrían apoyar el control reproductivo $(n=2)$. Se recibieron propuestas para castrarlas y luego liberarlas en áreas protegidas o comercializarlas como mascotas $(n=2)$. Algunos realizan una militancia activa para impedir acciones sobre las poblaciones establecidas y tienen apoyo de cientos de personas en blogs y redes sociales. Se muestran a favor de prevenir nuevas introducciones. Sin embargo, algunas de las propuestas de trasladarlas $(\mathrm{n}=4)$ para "salvaguardar" la vida de las ardillas (e.g., trasladarlas a bosques patagónicos o llevarlas a casas particulares) aumentan el riesgo de aparición de nuevos focos de invasión e ignoran posibles efectos sobre los ecosistemas $u$ otras especies. Se resalta la defensa de la vida de los animales y no se apoya su declaración como especie plaga.

\section{Vecinos}

Dentro de este grupo encontramos todo el espectro de opiniones y actitudes respecto de la ardilla de vientre rojo, desde positivas $(n=113)$ hasta negativas $(n=89)$. Los que consideran a la ardilla como "especie problema" lo hacen porque la asocian a los daños que sufren dentro de sus propiedades (visión más bien individualista, antropocéntrica y con una valoración dominante hacia la especie) o con el posible impacto sobre especies nativas o ecosistemas protegidos (con un enfoque ecocentrista). Muchos vecinos están a favor de "hacer algo" pero no hay acuerdo en cuanto a "¿qué hacer?" Algunos reclaman medidas de control y consideran que la presencia y expansión de ardillas en la zona debe ser atendida de forma urgente porque son una plaga. Otros reconocen que existe un problema pero rechazan cualquier tipo de manejo. La mayoría está a favor de las medidas de prevención y apoyan las campañas de difusión para que no se siga expandiendo. Sin embargo, algunos residentes están involucrados de manera directa en la captura y venta ilegal de ardillas, y otros expresan el deseo de tener ardillas donde aún no han llegado. La falta de denuncias sobre estas actividades que son bien conocidas por muchos vecinos se contradice con los reclamos de reparación a los responsables de haber introducido las ardillas en cada foco, que también suelen ser bien conocidos en el medio local. Por otro lado, los residentes que ven a las ardillas como "especie atractiva" no han sufrido daños causados por ardillas (o no los consideran suficientes), o no lo ven como justificativo como para tomar medidas de control. Muchos vecinos han convivido con ellas toda su vida por lo que a la especie le otorgan un valor simbólico y representativo de la localidad. Este enfoque zoocentrista y la valoración estética, naturalista y simbólica de la presencia de ardillas en la zona se traduce en actitudes en contra del sacrificio de individuos, aunque en algunos casos 
aceptarían el control reproductivo. Se registró una tendencia a una percepción positiva de la especie en ambientes urbanos mientras que la visión negativa fue predominante en áreas rurales. También observamos que al aumentar el tiempo de convivencia con las ardillas (e.g., mayor tiempo de introducción, disminuye la cantidad de personas indiferentes a su presencia y muchos vecinos que tenían una opinión positiva inicialmente cambian su parecer al ver los daños que les causa).

\section{Personal de áreas protegidas}

Los miembros de este grupo $(n=20)$ consideran a la ardilla como"especie problema" (valoración cientificista de la especie y del problema, con enfoque ecocentrista). Hay una genuina preocupación y estado de alerta frente al problema de las especies exóticas y mucho interés en conocer las características de la invasión para difundirlo a la comunidad. Están a favor de las medidas de prevención y alerta temprana hacia dentro y fuera de la reserva para evitar que la ardilla llegue a estos lugares, y también mostraron apoyo hacia acciones de control, utilizando métodos autorizados y consensuados.

\section{Funcionarios}

Existe diferente grado de conocimiento y compromiso con el tema por parte del personal de organismos gubernamentales. No obstante, la mayoría $(n=14)$ percibe a la ardilla de manera negativa, como especie problema, y hay una apertura a tener un enfoque ecocéntrico. En el caso de la localidad turística de La Cumbrecita, las autoridades locales consideran que la presencia de ardillas es positiva para el turismo, con una postura utilitaria. En las reuniones manifestaron voluntad de tomar medidas de prevención y control de la expansión de ardillas introducidas. Sin embargo, la gestión es lenta y compleja en parte por la falta de recursos humanos y logísticos para encarar acciones de manejo y por la falta de vinculación entre distintos estamentos (nacional/provincial/municipal, o sector productivo/medioambiente) pero también debido al grado de prioridad que se le asigna entre tantos conflictos pendientes de cada repartición y al costo político de controlar una especie carismática. En el nivel municipal sí se registran diferentes opiniones, propuestas y voluntad política de tomar una postura e iniciar acciones, lo cual dificultaría la coordinación de un plan de manejo en todos los municipios donde hay ardillas. El problema de la ardilla de vientre rojo no se considera una prioridad ni siquiera en Luján que, si bien es el municipio donde fue originalmente introducida y desde donde se envían ardillas a otras partes del país, enfrenta presiones de la comunidad para atender otras urgencias ambientales como la basura y la contaminación del agua. La falta de denuncias relacionadas con ardillas suele considerarse evidencia de que no son un problema relevante para los residentes, que a su vez no realizan denuncias por considerarlas inútiles. En varios municipios destacaron la necesidad de realizar una campaña informativa antes de encarar medidas de control. Se manifestó interés en actualizar la normativa vigente en torno a las especies exóticas, y a la ardilla de vientre rojo en particular, y se elaboró un decreto en la provincia de Buenos Aires, que incorpora a la ardilla de vientre rojo en el listado de especies perjudiciales, y una ordenanza en el partido de Luján que regula la captura y tráfico de ardillas y declara la necesidad de un plan de manejo integral de la especie.

\section{Docentes}

Dentro de este grupo hubo docentes y directivos con distintas percepciones respecto a la ardilla, tanto positivas como negativas, y en general mostraron una apertura a obtener más información y acercarse a una visión ecocéntrica del problema. Hubo interés y gran aceptación de la propuesta temática de los talleres, profundizando aspectos como la distinción entre exótico/nativo y doméstico/ silvestre, y resaltando la importancia de la responsabilidad como ciudadanos en cuanto a prevenir la creación de nuevos focos, denunciar actividades ilegales y promover la tenencia responsable de mascotas. Los docentes usaron en las aulas el material didáctico que les entregamos, abordaron el tema con sus alumnos y lograron un efecto multiplicador ya que fue compartido en sus hogares, o lo socializaron con otros cursos. Incluso, en algunos casos emprendieron proyectos áulicos más allá de nuestra propuesta didáctica. Estos proyectos incluyeron la elaboración de folletos, sitios web, programas radiales y hasta la elaboración de un proyecto de ordenanza para el municipio de Luján en torno a la problemática. En estos proyectos, los alumnos resaltaban la importancia de no dejarse engañar por la belleza de las ardillas y de frenar el comercio y traslado de animales silvestres. 


\section{CONSIDERACIONES GENERALES}

El rango de opiniones y actitudes descriptas respecto de la ardilla de vientre rojo es amplio, incluso dentro de algunos grupos como el que contiene a los residentes de zonas invadidas por ardillas que muestra todo el espectro de puntos de vistas. Ciertos factores como la experiencia directa con la especie y sus atributos, el área de residencia, el tiempo desde el establecimiento de ardillas en la zona y el conocimiento sobre la problemática asociada a las ardillas influyeron sobre la percepción de las personas que participaron en este estudio. Si bien hay variaciones según la historia de vida de cada persona y otros factores socio-demográficos, encontramos una percepción negativa mayoritaria y mayor predisposición para el control en residentes de áreas rurales que urbanas. Esto se relaciona con su dependencia con actividades productivas que pueden ser afectadas por especies silvestres pero también con hábitos ya incorporados, como la caza y el control de especies que causan o pueden causar daños. No todos los animales silvestres son vistos como "pestes" pero hay un consenso implícito en que tienen que ser controlados (Oppel et al. 2011). Esto por un lado facilita realizar acciones de control pero por otro dificulta que se incorporen a programas de control integrados y autorizados. En zonas urbanas suele encontrarse un menor registro de daños y mayor diversidad cultural y conociminetos que se traduce en un amplio rango de valoraciones, actitudes y conductas respecto de la fauna y de la naturaleza en general (Kellert 1996). También las características particulares del lugar influyen sobre la valoración de la especie, como se observó en la localidad La Cumbrecita, Córdoba, dado que es considerada una atracción turística, algo que causó su introducción en el lugar y genera resistencia a iniciar acciones tendientes al control o erradicación del foco.

El tiempo transcurrido desde la introducción de la especie en el lugar influye sobre la percepción y actitud de las personas respecto de la presencia de ardillas ya que incrementa el contacto directo con la especie y disminuye la cantidad de personas indiferentes a su presencia; de esta manera, aumenta tanto la proporción de residentes que reclaman acciones de manejo por los daños registrados como la proporción de residentes que valoran la presencia de ardillas en su ambiente cotidiano. Lo mismo ocurre con otras especies introducidas ya que se observa una relación entre la memoria histórica de los residentes y el reconocimiento de una especie como exótica (García Llorente et al. 2008; Schuttler et al. 2011). Cuando las introducciones fueron muy lejanas en el tiempo, las personas no las reconocen como especies introducidas $y$ desconocen los daños que provocan, como ocurre con el castor (Castor canadiensis) en tierra del Fuego (Schuttler et al. 2011) o especies que se consideran "naturalizadas" como la liebre (Lepus capensis) o inclusive el ganado. En nuestro país y en Sudamérica en general, existe un desconocimiento del pasado biológico del lugar de gran parte de los residentes, en particular jóvenes, que inclusive pueden conocer, proteger y valorar más a algunas especies introducidas que a las nativas, como ocurre con el pino (Pinus spp.), el ciervo colorado (Cervus elaphus) o la trucha (Salmo spp.) en la Patagonia (Speziale et al. 2012).

Los atributos de la especie introducida (en cuanto a su apariencia y reputación) influyen sobre la percepción y actitud de las personas independientemente del impacto que cause, por lo que la percepción de una especie introducida es cultural y organismodependiente (Simberloff 2011). En general hay mayoratracciónhaciaanimalesevolutivamente más cercanos a humanos y más rechazo hacia los más lejanos, como invertebrados (Batt 2009). Dentro de los mamíferos, suele haber grupos más rechazados como los carnívoros (Schuttler et al. 2011) y hay especies "odiadas" como las ratas (Rattus spp.) y otras "amadas", como pueden ser las ardillas para algunos pobladores de nuestro estudio. Este grado de aceptación o rechazo a ciertas especies influyen sobre el apoyo a planes de control de EEI, como suele observarse al comparar el escaso apoyo a la remoción de aves o mamíferos frente a la aceptación del control de invertebrados, y también una mayor aceptación del uso de métodos químicos para remover plantas que animales (Bremner \& Park 2007; Fraser 2001, 2006). Justamente, el valor carismático de la ardilla gris (Sciurus carolinensis) hizo fracasar su erradicación en Italia por la oposición activa de los grupos defensores de animales (Bertolino \& Genovesi 2003; Perry 2004; Selge et al. 2011). De alguna manera, el carisma de la especie incrementa el nivel de daños o conflicto que es necesario demostrar para apoyar medidas de control que involucren sacrificio de animales, como también se ha informado para el caso de 
caballos cimarrones (Equus ferus caballus) en áreas protegidas de la provincia de Buenos Aires (Scorolli 2002), y castores o visones (Neovison vison) en Tierra del Fuego (Schuttler et al. 2001; Sharp et al. 2011).

Algunos estudios destacan cómo el conocimiento previo de los daños que ocasionan las EEIy delos programas de manejo que se implementarán y sus fundamentaciones incrementan la aceptación por parte de la comunidad (Fraser 2006; Bremner \& Park 2007; Sharp et al. 2011). Incluso la participación de distintos grupos sociales en las decisiones de manejo se está volviendo cada vez más importante para el éxito de las iniciativas de conservación (Fischer \& Young 2007). Las actividades de capacitación y difusión que realizamos promovieron que muchas personas analizaran el caso de la ardilla de vientre rojo bajo un marco conceptual más amplio sobre invasiones biológicas y con información actualizada sobre daños, riesgos, vías de expansión, y teniendo en cuenta la complejidad de cualquier plan de control. El acceso a nueva información abrió las puertas a la generación de cambio de actitudes frente a la responsabilidad como ciudadanos y revalorización del ecosistema local. Los factores antes mencionados indican que las opiniones y actitudes respecto de las EEI se forjan mediante procesos complejos, dinámicos y polifacéticos, que subrayan la necesidad de considerar a las invasiones biológicas bajo marcos conceptuales tanto biológicos como sociales. Los desafíos para hacer frente a esta problemática están puestos en articular la conservación de la biodiversidad con otras preocupaciones sociales, para lo cual es necesario conocer y considerar cómo una sociedad percibe las invasiones y favorecer la interacción de los distintos actores sociales interesados (Simberloff 2011).

El manejo de una EEI requiere considerar cómo afectará a los habitantes de un lugar y a las actividades humanas (Oppel et al. 2011), algo que resulta clave cuando se trabaja con especies introducidas que son carismáticas y que se han establecido en áreas urbanas y rurales, donde las acciones de manejo deben ser coordinadas dentro de tierras públicas y privadas. Para esto es necesario plantear alternativas de manejo diferentes para cada zona o región invadida con una visión integral del problema. De esta manera, para cada foco de invasión de ardillas sería posible planificar la erradicación o el control según el grado de expansión y abundancia de ardillas y las características socio-económicas y políticas de la zona. Al mismo tiempo, sería necesaria una estrategia nacional de prevención para frenar la generación de nuevos focos de invasión por liberación de individuos, así como un plan de contención en ciertas zonas donde las ardillas están cercanas a zonas valiosas desde el punto de vista de la conservación, por ejemplo en la zona del Delta del Río Paraná. En este sentido se dieron los primeros pasos dando información sobre el problema, iniciando medidas de prevención, impulsando la actualización de la legislación vigente, y evaluando posibles acciones por parte de organismos de gestión. Las diferentes opiniones y actitudes de distintos grupos sociales y la oposición marcada a los planes de control de algunos grupos, que podrían llevar al fracaso estos planes, muestran la necesidad de mejorar la comunicación y vínculo entre organismos de gestión, investigadores y ciudadanía. Al mismo tiempo habría que iniciar pruebas piloto que contemplen la complejidad biológica y social para evaluar la factibilidad de acciones de manejo concretas en zonas particulares.

Agradecimientos: Varios estudiantes colaboraron en las actividades de capacitación, difusión y entrevistas: $\mathrm{S}$. Almada Chavez, L. Messetta, Y. Bobadilla, D. Acosta, N. Mufato, M. Herztiken y A. Troyelli. E. Barbetti, E. Viola y N. Gorla colaboraron en los talleres de capacitación docente. Agradecemos a todas las personas que participaron en los talleres, charlas, entrevistas o que se comunicaron con nosotros en relación a la problemática de la invasión de ardillas. Los comentarios de dos revisores anónimos contribuyeron a una mejor presentación del trabajo. La Universidad Nacional de Luján financió este trabajo mediante subsidios de investigación y de extensión.

\section{BiBLIOGRAFÍA}

ApriLe, G \& D Chicco. 1999. Nueva especie exótica de mamífero en la Argentina: la ardilla de vientre rojo (Callosciurus erythraeus). Mastozoología Neotropical, 6:7-14.

BATT, S. 2009. Human attitudes towards animals in relation to species similarity to humans: a multivariate approach. Bioscience horizons, 2:180-190.

Benitez, VV; S Almada Chavez; AC Gozzi; ML Messetta; et AL. 2013. Invasion status of Asiatic red-bellied squirrels in Argentina. Mammalian Biology, 78:164-170.

Bertolino, S \& P GenOvesi. 2003. Spread and attempted eradication of the grey squirrel (Sciuruscarolinensis) in Italy, and consequences for the red squirrel (Sciurus vulgaris) in Eurasia. Biological Conservation, 109:351-358.

Bobadilla, SY; P ZubelzÚ; VV BenITEZ \& ML GUICHÓN. 2012. Mamíferos introducidos como dispersores de semillas en la Región Pampeana: ¿la ardillas de vientre rojo (Callosciurus erythraeus) facilita el establecimiento de 
vegetación exótica? Póster. II Congreso Latinoamericano de Mastozoología y XXV Jornadas Argentinas de Mastozoología, Buenos Aires, Argentina.

Bremner, A \& K PArk. 2007. Public attitudes to the management of invasive non-native species in Scotland. Biological Conservation, 139:306-314.

Fischer, A \& JC Young. 2007. Understanding mental constructs of biodiversity:Implications for biodiversity management and conservation. Biological Conservation, 136:271-282.

FRASER, W. 2001.Introduced wildlife in New Zealand: a survey of general public views. Landcare Research Science Series No 23. ManaakiWhenua Press, Canterbury, New Zealand.

Fraser, A. 2006. Public Attitudes to Pest Control: A Literature Review. Department of Conservation, Wellington, New Zealand. Pp. 36.

García-Llorente, M; M López; JA González; P Alcorlo \& C MONTES. 2008. Social perceptions of the impacts and benefits of invasive alien species: Implications for management. Biological Conservation, 141:2969-2983.

GLAVES, P. 2009. Invasive, alien and problem species in urban areas: some social, political and philosophical dimensions $P p$. 53-59 en: Rotherham, ID (ed.). Exotic and invasive plants and animals, International Urban Ecology Review 4 (1). Wildtrack Publishing, Sheffiled, Reino Unido. Pp. 127.

Gozzi, AC; ML Guichón; VV Benitez \& M LaReschi. 2013a. Arthropod parasites of the red-bellied squirrel Callosciurus erythraeus (Rodentia: Sciuridae) introduced into Argentina. Medical and Veterinary Entomology, 27: 203-208.

Gozzi, AC; ML Guichón; VV Benitez; GN Romero; C AUTERI \& B BRIHUEGA. 2013b. First isolation of Leptospira interrogans from the introduced arboreal squirrel Callosciurus erythraeus (Rodentia: Sciuridae) introduced in Argentina. Wildlife Biology, 19:1-7.

Guichón, ML; M Bello \& L Fasola. 2005. Expansión poblacional de una especie introducida en la argentina: la ardilla de vientre rojo Callosciurus erythraeus. Mastozoología Neotropical, 12(2):189-197.

GUICHÓN, ML \& CP DONCASTER. 2008. Invasion dynamics of an introduced squirrel in Argentina. Ecography, 31: 211-220.

IUCN. 2000. Guidelines for the Prevention of Biodiversity Loss Caused by Alien Invasive Species. IUCN. Gland, Switzerland. Pp. 14.

Kellert, SR. 1996.The Value of Life. Island Press, Washington, D.C. USA.

LARSON, BMH. 2005. The war of the roses: demilitarizing invasion biology. Front. Ecol. Environ., 3:495-500.

LodGe, DM \& K SHRADER-FrechetTe. 2003. Nonindigenous Species: Ecological Explanation, Environmental Ethics, and Public Policy. Conservation Biology, 17:31-37.

LundBerG, A. 2010. Conflicts between perception and reality in the management of alien species in forest ecosystems: a Norwegian case study. Landscape Research, 35:319-338.

MaCK, RN; D SimberLofF; WM LondSDALE; H Evans; M Clout; ET AL. 2000. Biotic invasions: causes, epidemiology, global consequences, and control. Ecological Applications, 10:689-710.

MESSETTA, ML. 2012. Impacto de la introducción de la ardilla de vientre rojo (Callosciurus erythraeus) sobre las aves en la Región Pampeana. Tesis de Licenciatura, Universidad Nacional de Luján. Argentina.

McNeely, JA. 2011. Xenophobia or conservation: some human dimensions. Pp. 19-36 en: Rotherham, ID \& RA Lambert (eds.). Invasive and Introduced Plants and Animals - Human Perceptions, Attitudes and Approaches to Management. Earthscan. London, UK.

Oppel, S; BM Beaven; M Bolton; J Vickery \& TW Bodey. 2011. Eradication of Invasive Mammals on Islands Inhabited by Humans and Domestic Animals. Conservation Biology, 25:232-240.

Perry, D. 2004. Animal Rights and Environmental Wrongs: The Case of the Grey Squirrel in Northern Italy. Essays in Philosophy, 5(2):26.

Pimentel, D; $\mathrm{R}$ Zuniga \& $\mathrm{D}$ Morrison. 2005. Update on the environmental and economic costs associated with alien-invasive species in the United States. Ecological Economics, 52:273-288.

Salazar Barragan, DM \& CA Serna Mendoza. 2006. Ética, medioambiente y economía. Persona y Bioética, 10(26). Universidad La Sabana, Cundinamarca, Colombia. Pp. 8-34.

SCOROLli, AL. 2002. Salvajes los caballos de Sierra de la Ventana. Vida Silvestre, 81:18-19.

Selge, S; A Fisher \& R Van der Wal. 2011. Public and professional views on invasive non-native species - A qualitative social scientific investigation. Biol. Conserv., 144(12):3089-3097.

SHARP, RL; R LiNCOLN; B LARSON \& GT GREEN. 2011 Factors influencing public references for invasive alien species management. Biological Conservation, 144:2097-2104.

SCHÜtTLER, E; R RozZI \& K JAX. 2011. Towards a societal discourse on invasive species management: A case study of public perceptions of mink and beavers in Cape Horn. Journal for Nature Conservation, 19:175-184.

SIMBERLOFF, D. 2000. Nonindigenous species: a global threat to biodiversity and stability. En: Raven, P \& T Williams (eds). Nature and Human Society: the Quest for a Sustainable World. National Academy Press. Washington. USA.

SimberlofF, D. 2003. Confronting introduced species: a form of xenophobia? Biological Invasions, 5:179-192.

SimberLOFF, D. 2011. The rise of modern invasion biology and American attitudes towards introduced species. Pp. 121-135 en: Rotherham, ID \& RA Lambert (eds.). Invasive and Introduced Plants and Animals - Human Perceptions, Attitudes and Approaches to Management. Earthscan, London. UK.

SimberlofF, D; JL Martin; P Genovesi; V Maris; DA WardLE; ET AL. 2013. Impacts of biological invasions: what's what and the way forward. TREE, 28:58-66.

Speziale, KL; SA Lambertucci; M Carrete \& JL Tella. 2012. Dealing with non-native species: what makes the difference in South America? Biol. Invasions, 14: 1609-1621.T 